\title{
Histogram Equalization: A Strong Technique for Image Enhancement
}

\author{
Ravindra Pal Singh and Manish Dixit \\ Dept. of Comp. Science/IT \\ MITS Gwalior, 474005 India \\ ravindramits13@gmail.com,dixitmits@gmail.com
}

\begin{abstract}
Generally for improving contrast in digital images, $H E$ is the method that commonly used but in result it gives unnatural artifacts like intensity saturation, over-enhancement and noise amplification. To overcome these problems there was a need to partition the image histogram, at first image histogram was partitioned into two parts and then different transformation functions were applied on each partition. After that image histogram was partitioned into many partitions and same process was applied with some additional features. DHE is the multi histogram method and CLAHE is the extension of AHE. These methods are compared to HE and found that both methods give better result than HE but DHE method also gives better result than CLAHE.
\end{abstract}

Keywords: Image Processing, Contrast Enhancement, Histogram Processing etc.

\section{Introduction}

Image enhancement is a process to remove the unwanted distortion due to deterioration in contrast, unwanted noise, improper intensity saturation, blurring effect etc, and determine the hidden information that are contained in images [1,3]. And image enhancement gives better visually images that are given as input to various digital image processing applications. At present, in this paper we only concentrated on the contrast enhancement of digital images that can be used in different applications like biometric analysis, pattern identification, facial acknowledgement, medical fields, and in consumer electronics applications. Contrast is an image element that could be defined as a ratio between the highest and the lowest pixel intensities of an image. In image processing system contrast enhancement is a strenuous task to be done as well as it is very difficult to be obtained a good contrast image. There are several descriptions for an image to have poor contrast: due to the poor quality of the used imaging device. The images usually suffer from poor image quality, degradation in contrast and happening of shading and artifacts, due to lack in centering pixel intensity, poor lightening, specimen spotting and other factors that can be manipulated by image enhancement methods. Medical images, palm images, satellite images, remote sensing images, and electron-microscopy images even real life photographic pictures suffer from degradation in contrast [2, 3] which leads to enhance the contrast. Histogram of images provides a description of the image appearance globally. Histogram equalization and linear contrast manipulation are very frequently used techniques for improving the degraded image contrast. But both the methods are not suitable for retaining the natural contrast rather that they may suffer from unnatural contrast and some unwanted artifacts. Histogram gives huge information about image attributes and hence Histogram modeling through a spatial domain technique is of great importance in Digital Image Processing and has been implemented on various platforms. In this paper contrasting types of histogram equalization mechanisms are studied and compared to each other. Dynamic histogram equalization (DHE) is a multihistogram equalization method which is better than bi-histogram equalization method and 
Contrast limited adaptive histogram equalization (CLAHE) is a clip histogram equalization technique that is better than classical AHE. The CLAHE approach is an extended version of adaptive histogram equalization method with some additional parameters that are used to overcome the problem with adaptive histogram equalization.

\section{Histogram Equalization}

Histogram equalization is a spatial domain method that produces output image with uniform distribution of pixel intensity means that the histogram of the output image is flattened and extended systematically [2, 4]. This approach customarily works for image enhancement paradigm because of its simplicity and relatively better than other traditional methods. We acquire the probability density function (PDF) and cumulative density function (CDF) via the input image histogram. Apply these two functions PDF and CDF for replacing the input image gray levels to the new gray levels, and then we generate the processed image and histogram for the resultant image. And when we discriminate input image histogram with the processed image histogram we found that the gray level intensities are stretched and depressed systematically. Consequently, we obtain that the histogram of the output image is systematically distributed. Yet, this accords the over enhancement in images above the actual gray scale span. During histogram equalization approach the mean brightness of the processed image is always the middle gray level without concerning of the input mean. This procedure is not very convenient to be enforced in consumer electronics, such as television, by the reason of that the method tends to introduce irrelevant visual deterioration like the concentration effect. The particular explanation for this issue is to conquer this weakness is by perpetuating the mean brightness of the input image indoor the output image. Figure 2.1 shows an illustrating example of using Histogram Equalization (HE) for image contrast enhancement.

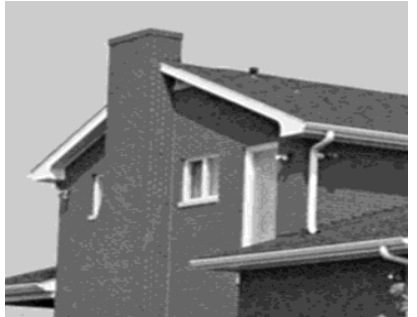

(a)

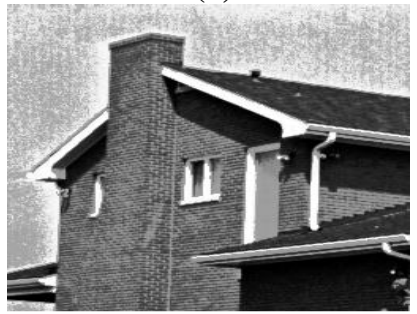

(c)

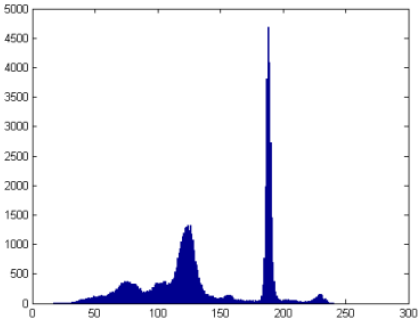

(b)

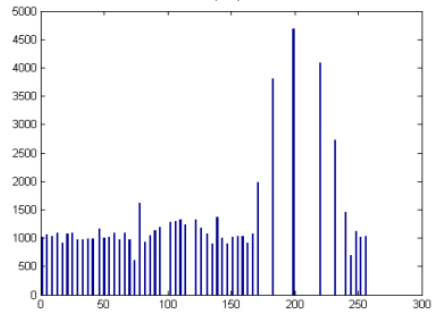

(d)

Figure 2.1 Illustration of HE: (a) Input image, (b) Histogram of the output image, (c) Output image by the HE method, (d) Histogram of the output image

\section{Dynamic Histogram Equalization}

Dynamic Histogram Equalization (DHE) was essentially popularized in 2007 by Wadud et al. [8], to eliminate the influence of higher histogram components on lower histogram components in the image histogram and to regulate the amount of spreading of gray levels for objective enhancement of the image appearance by using local minima 
separation of histogram. DHE displays continuous and better enhancement of the image than the traditional paradigm. Withal, the DHE oversight the mean brightness perpetuation and influences to intensity saturation artifacts [9]. DHE technique has overcome the drawbacks of histogram equalization and has shown a better brightness preserving and contrast enhancement than HE. DHE reinforces the image beyond making any destruction in image particulars. However, if user is not satisfied, may control the extent of enhancement by adjusting only one parameter. Besides, DHE is transparent and computationally adequate which makes it easy to implement and can be operated in real time systems. The flowchart for the DHE algorithm is shown in Figure 3.1.

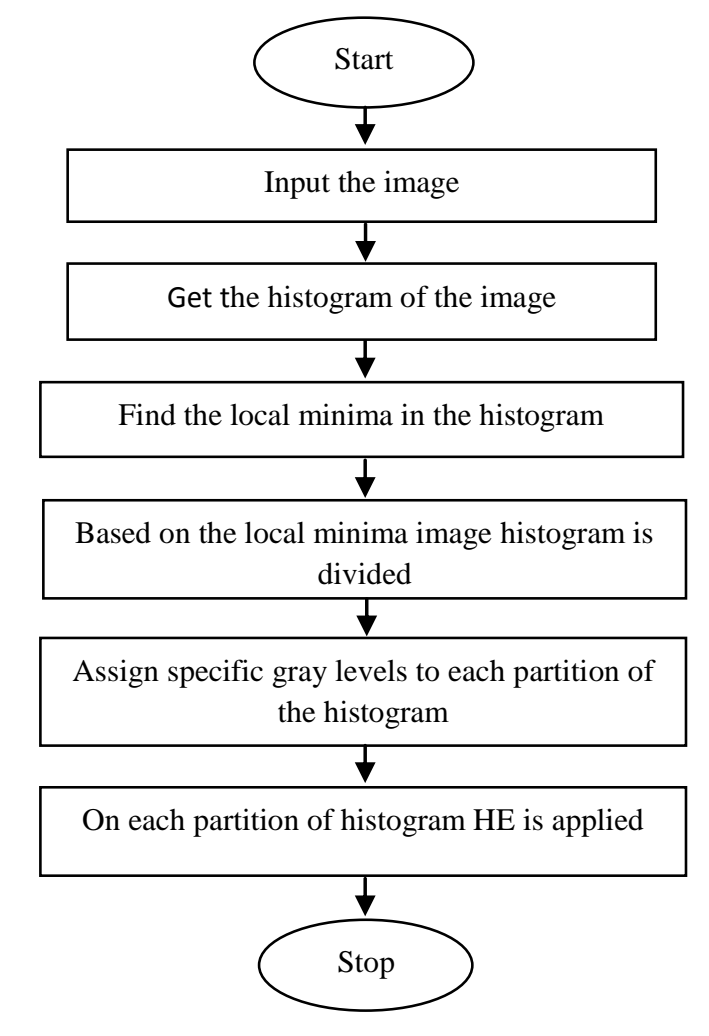

Figure 3.1. Flow Diagram for DHE Method

DHE separates the histogram depends on local minima. Formally, it implements a onedimensional smoothing filter on the histogram to dispose meaningless minima. Then it makes sub-histograms taking the portion of histogram that falls between two local minima. Figure 3.2 shows an illustrating example of using Dynamic Histogram Equalization (DHE) for image contrast enhancement.'

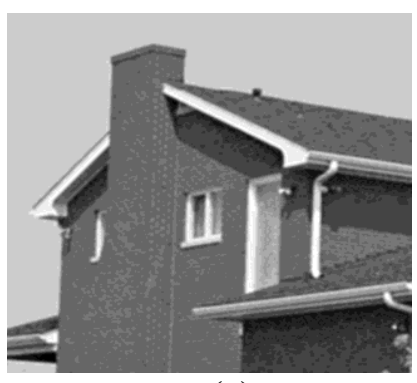

(a)

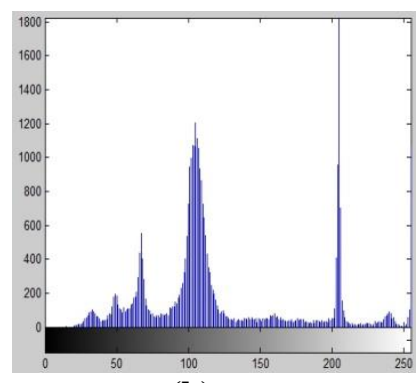

(b) 


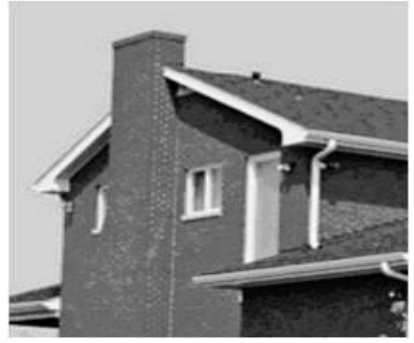

(c)

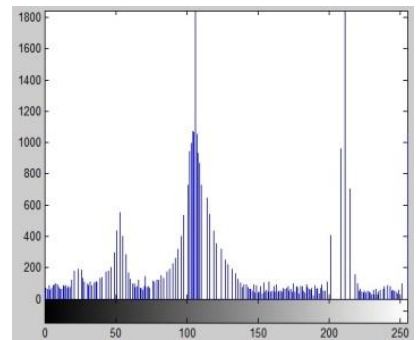

(d)

Figure 3.2. Illustration of DHE: (a) Input image, (b) Histogram of the output image, (c) Output image by the DHE method, (d) Histogram of the output image

\section{Contrast Limited Adaptive Histogram Equalization}

In few cases when grayscale distribution is extremely localized, it may not be enticing to transform low contrast images by Histogram Equalization approach. Therefore, in these cases aligning the curve may include segments with high slopes means two grayscale might be mapped to significantly different grayscales. This issue can be resolved through Histogram Equalization by limiting the contrast and the technique used for this condition is known as CLAHE (Contrast Limited Adaptive Histogram Equalization). Whereas on applying AHE, if the refined region has approximately small intensity then in that region the noise get more enhanced but there may be some artifacts on that region. CLAHE is the suitable method that is used to limit those artifacts. CLAHE promotes on minuscule image blocks and each block's contrast is being enhanced, so that the processed histogram region approximately matches the histogram specified by a distribution that may be any of Binomial, Gaussian, Poisson or Rayleigh. The neighboring blocks are jointed using bilinear interpolation to wipe out artificially induced perimeters. The contrast in homogenous area can be explained to evade strengthening any noise. Originally it was advanced for medical imaging and verified to be successful for the enhancement of low contrast images such as portal films [6]. To control the quality of the image CLAHE uses block size and clip limit as the parameters and these may have some default value sometimes these values are chosen by users. And when a user selects parameters values randomly, the results of the CLAHE would be degraded than HE approach [4,7]. The CLAHE introduced clip limit to overcome the noise problem. Before calculating the CDF, the CLAHE limits the amplification by clipping the histogram at a predefined value. This limits the slope of the CDF and therefore of the transformation function. Clip limit is a value based on the histogram is clipped, depends on the normalization of the histogram and thereby on the size of the neighborhood region [5]. The algorithm for CLAHE is shown in Figure 4.1. 


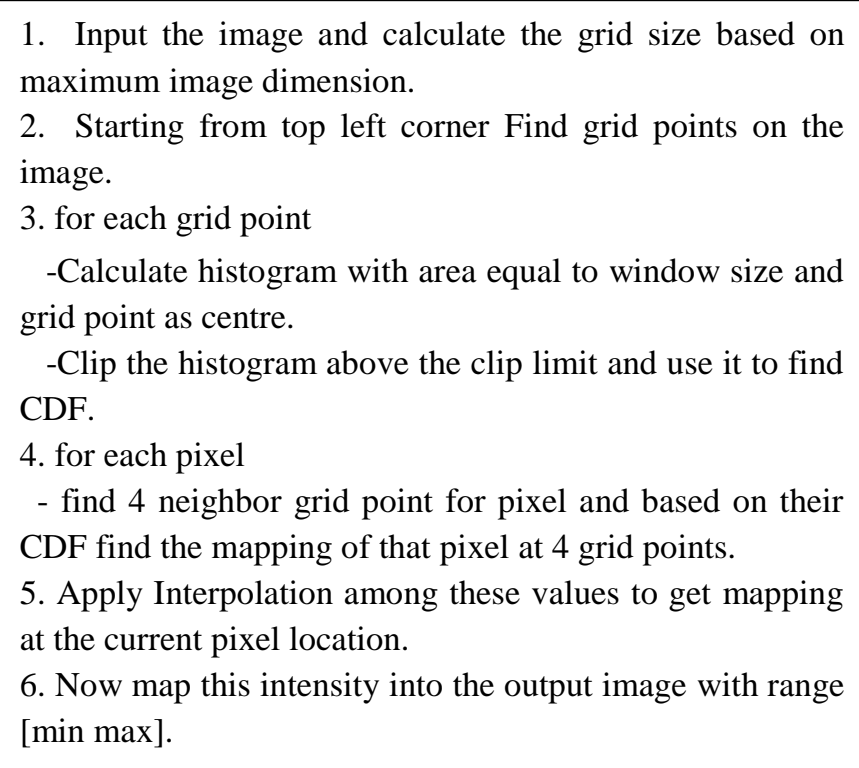

Figure 4.1. Algorithm for CLAHE Method

In the algorithm if a window size is not specified choose the grid size as the default window size and each grid point is separated by grid size pixels. After calculating the mappings for each grid point, steps 4 to 6 for each pixel in the input image are repeated. Clipping the histogram itself is not quite straight forward because the excess after clipping has to be redistributed with the new bins that may increments the level of the clipped histogram. Therefore the clipping should be executed at a level lower than the detailed clip level so that after reorganization, the maximum histogram level is equal to the clip level. Figure 4.2 shows an illustrating example of using Contrast Limited Adaptive Histogram Equalization (CLAHE) for image contrast enhancement.

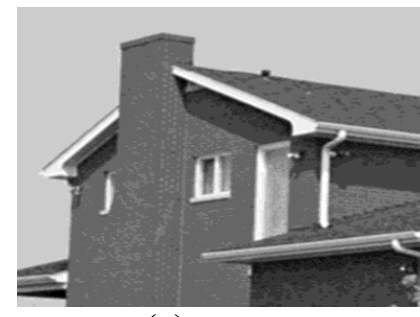

(a)

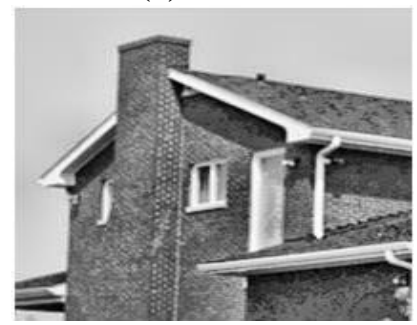

(c)

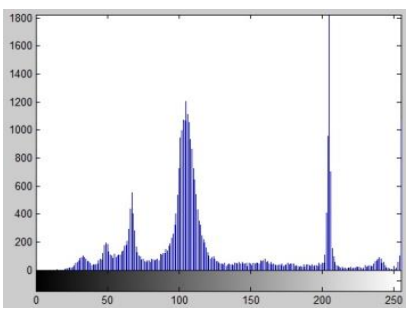

(b)

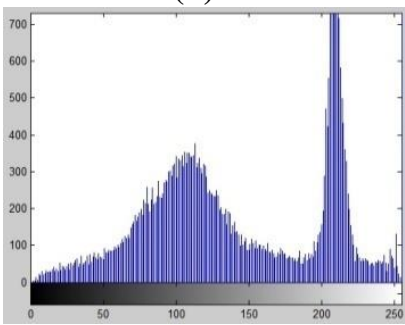

(d)

Figure 4.2 Illustration of CLAHE: (a) Input image, (b) Histogram of the output image, (c) Output image by the CLAHE method, (d) Histogram of the output image 


\section{Results and Discussion}

The following methods for contrast enhancement are applied on various images and the resultant images formed by these methods are compared statistically with PSNR (Peak Signal to Noise Ratio) and NAE (Normalized Absolute Error). The PSNR values and NAE values for output images are stored in tabular form and Shown in Table 5.1 and Table 5.2 respectively. And the graphical illustration of PSNR and NAE are shown by Figure 5.2 and Figure 5.3 respectively.

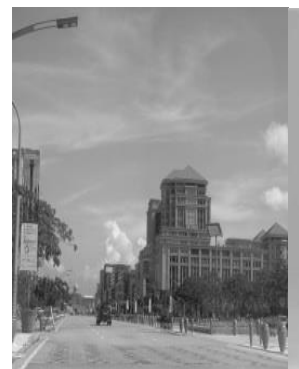

(a)

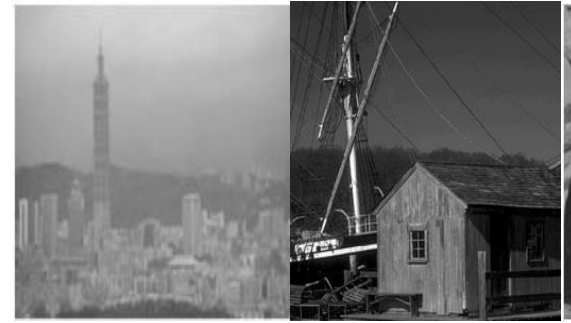

(d) (e)

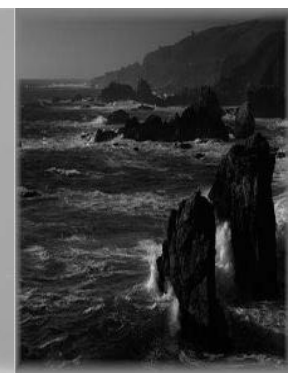

(c)

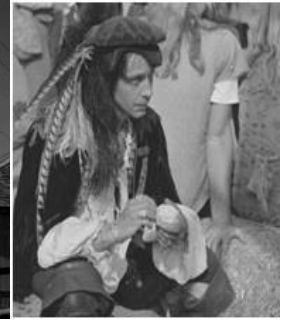

(f)

Figure 5.1. Illustration of input images: (a).Road- side building (b)Girl (c)Beach (d)Building (e)House (f)Pirate

Below in Table 5.1, the PSNR values for output images are tabulated. From these values we see that only for (d).building and (b). girl image CLAHE gives higher PSNR value than the HE and DHE. But for the images (a). Road-side building (c). Beach (e). House and (f). Pirate, the DHE gives higher PSNR. value. And when we plot graph according to the PSNR values

Table 5.1. The PSNR Values for Various Contrast Enhancement Techniques

\begin{tabular}{|c|c|c|c|}
\hline Image Name & HE & DHE & CLAHE \\
\hline Building & 13.505 & 14.925 & $\mathbf{2 1 . 3 2 0}$ \\
\hline Road-side building & 15.124 & $\mathbf{2 2 . 5 1 7}$ & 20.641 \\
\hline Girl & 11.444 & 19.682 & $\mathbf{2 3 . 1 6 0}$ \\
\hline Beach & 9.268 & $\mathbf{1 6 . 3 1 7}$ & 13.542 \\
\hline House & 10.800 & $\mathbf{1 9 . 7 5 6}$ & 15.342 \\
\hline Pirate & 18.715 & $\mathbf{2 1 . 7 4 2}$ & 17.717 \\
\hline
\end{tabular}

for the Output images, and then except for two images the graph for DHE looks better than HE and CLAHE methods. Therefore, we can say that DHE method gives better result than HE and CLAHE. 


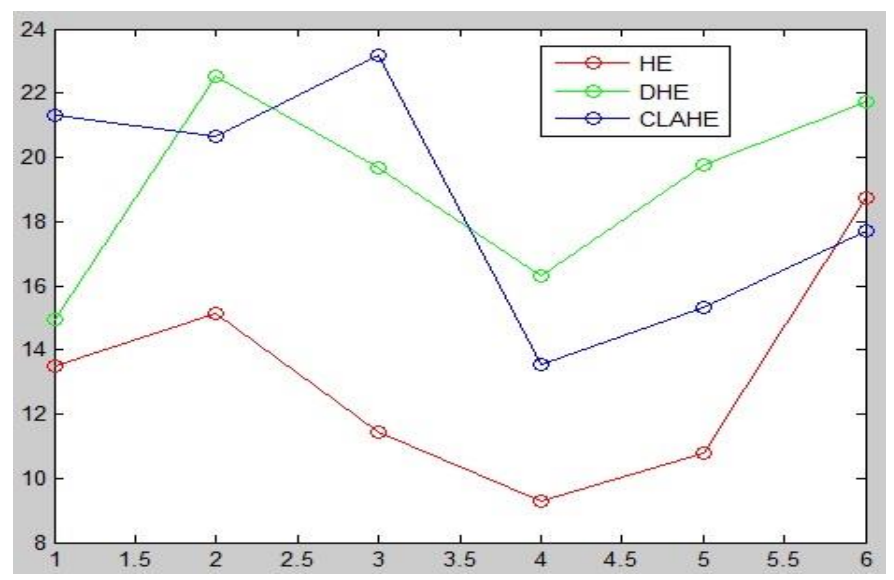

Figure 5.2. Illustration of the PSNR Graph for Various Techniques for Contrast Enhancement

Table 5.2. The NAE Values for Various Contrast Enhancement Techniques

\begin{tabular}{|c|c|c|c|}
\hline Image Name & HE & DHE & CLAHE \\
\hline Building & 0.2721 & 0.2585 & $\mathbf{0 . 1 0 5 2}$ \\
\hline Road-side building & 0.2895 & $\mathbf{0 . 0 9 0 6}$ & 0.1271 \\
\hline Girl & 0.3717 & 0.1421 & $\mathbf{0 . 0 7 8 9}$ \\
\hline Beach & 1.5832 & $\mathbf{0 . 4 7 1 4}$ & 0.8852 \\
\hline House & 0.8874 & $\mathbf{0 . 1 6 8 2}$ & 0.5196 \\
\hline Pirate & 0.2041 & $\mathbf{0 . 1 6 2 3}$ & 0.2294 \\
\hline
\end{tabular}

NAE values for output images are tabulated. From these values we see that only for (d).building and (b). girl image CLAHE gives lower NAE value than the HE and DHE. But for the images (a). Road-side building (c). Beach (e). House and (f). Pirate, the DHE gives lower PSNR value. And when we plot graph according to the NAE values for the images, then except for two images the graph for DHE looks better than HE and CLAHE methods. Therefore, we can say that DHE method gives better result than HE and CLAHE.

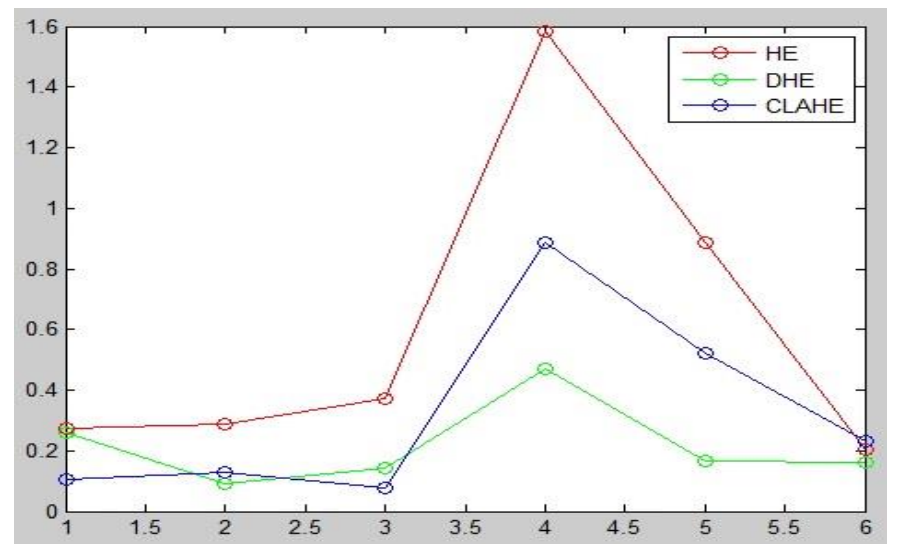

Figure 5.3. Illustration of the NAE Graph for Various Techniques for Contrast Enhancement 


\section{Conclusion}

$\mathrm{HE}$ is a simple and effective contrast enhancing technique. But it significantly changes the image brightness. On the other hand CLAHE and DHE both methods increases the contrast more than HE. CLAHE introduces large changes in the pixel intensity, which leads to some processing artifacts and affect the decision making process. By the values of PSNR and NAE the DHE method gives better result than HE and CLAHE. Operating on the global statistics of images DHE is computationally more efficient than CLAHE. So, DHE is more efficient method than the HE and CLAHE but the main drawback with DHE is that it is not good with preserving brightness of the images. Therefore, while using DHE at last we have to normalize the brightness of the image.

\section{References}

[1] R. C. Gonzalez and R. E. Woods, "Digital Image Processing", 2nd edition, Prentice Hall, (2002).

[2] R. C. Gonzalez and R. E. Woods, "Digital image processing," Third Edition, Prentice Hall.

[3] S. Jayaraman, S. Esakkirajan, and T. Veerakumar, "Digital image processing," Third Edition, Mcgraw Hill education.

[4] A. K. Jain,"Fundamentals of Digital Image Processing Englewood Cliffs," NJ: Prentice Hall, (1989).

[5] R. Garg, B. Mittal, and S. Garg, "Histogram Equalization Techniques for Image Enhancement," International Journal on Electronics \& Communication Technology, vol. 2, no. 1, (2011), pp. 107-111.

[6] K. Zuiderveld, "Contrast Limited Adaptive Histogram Equalization", Academic Press, Cambridge, (1994).

[7] M. Kaur, J. Kaur, and J. Kaur, "Survey of contrast enhancement techniques based on histogram equalization", International Journal of Advanced Computer Science and Applications, vol. 2, no. 7, (2011), pp. 137-141.

[8] M. A. A. Wadud, M. H. Kabir, M. A. A. Dewan and O. Chae, "A Dynamic Histogram Equalization for Image Contrast Enhancement", IEEE Transactions on Consumer Electronics, vol. 53, no. 2, (2007) May, pp. 593-600.

[9] A. Raju, G. S. Dwarakish and D. V. Reddy, "A Comparative Analysis of Histogram Equalization based Techniques for Contrast Enhancement and Brightness Preserving”, International Journal of Signal Processing, Image Processing and Pattern Recognition, vol. 6, no. 5, (2013), pp. 353-366. 